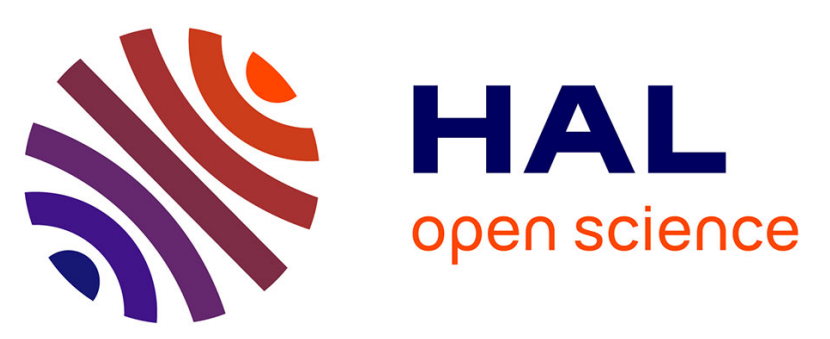

\title{
Etudes racinaires au sein du programme "croissance" de l'INRA (Quercus petraea et Pinus pinaster)
}

\author{
Francis Colin, Frédérique Danjon, Léon Wehrlen
}

\section{To cite this version:}

Francis Colin, Frédérique Danjon, Léon Wehrlen. Etudes racinaires au sein du programme "croissance" de l'INRA (Quercus petraea et Pinus pinaster). Revue forestière française, 1995, 67 (S), pp.165-172. $10.4267 / 2042 / 26709$. hal-03444433

\section{HAL Id: hal-03444433 \\ https://hal.science/hal-03444433}

Submitted on 23 Nov 2021

HAL is a multi-disciplinary open access archive for the deposit and dissemination of scientific research documents, whether they are published or not. The documents may come from teaching and research institutions in France or abroad, or from public or private research centers.
L'archive ouverte pluridisciplinaire HAL, est destinée au dépôt et à la diffusion de documents scientifiques de niveau recherche, publiés ou non, émanant des établissements d'enseignement et de recherche français ou étrangers, des laboratoires publics ou privés. 


\title{
ÉTUDES RACINAIRES \\ AU SEIN \\ DU PROGRAMME "CROISSANCE" \\ DE L'INRA \\ (Quercus petraea et Pinus pinaster)
}

\author{
F. COLIN - F. DANJON - L. WEHRLEN *
}

\section{ANTÉCÉDENTS ET MOTIVATIONS ACTUELLES}

Le Département des Recherches forestières de I'INRA a, de tout temps, mené des études sur les systèmes racinaires des arbres forestiers suivant des objectifs variables, par exemple:

- la mise au point des meilleures techniques d'élevage en pépinière et en plantation (par exemple, Riedacker, 1978) ;

- la recherche des causes de dépérissement (Guillaumin et al., 1985);

- l'évaluation des potentialités de croissance aérienne et racinaire des plantations réalisées en sols difficiles (engorgés d'eau temporairement, secs temporairement) (Lévy, 1968; Belgrand, 1983) ;

- la compréhension des modalités de prélèvement de l'eau et des éléments minéraux dans les sols forestiers (Bréda et al., 1993) ;

- les études sur le fonctionnement des taillis (Bédéneau et Auclair, 1989);

- la mise au point de méthodes de description, d'analyse et de modélisation de la croissance des jeunes plants (Colin-Belgrand et al., 1989).

Le "programme croissance" du Département des Recherches forestières s'est pour l'instant attaché à définir les normes de sylviculture des grandes essences forestières. II a utilisé pour cela les données recueillies principalement dans ses dispositifs expérimentaux anciennement établis. L'effet de nouveaux scénarios de sylviculture (plus intensifs, ou faisant intervenir le mélange d'espèces, ou bien encore tenant compte des larges espacements de plantation actuellement préconisés sur anciennes terres agricoles) ne peut être évalué à partir de ces dispositifs seuls. Les informations ayant permis initialement la définition des normes, et des données récentes recueillies sur un nombre faible d'individus ont permis d'établir des modèles de croissance. Après une phase de validation, ils devraient permettre de rendre compte des comportements de croissance dans des situations nouvelles.

* avec la collaboration technique de $Y$. Lefèvre pour les descriptions de sol de Nancy et de $\mathrm{Y}$. Ledore pour les dessins. 


\section{F. COLIN - F. DANJON - L. WEHRLEN}

Faute de données, ces modèles ne prennent pas en compte les systèmes racinaires. En leur absence, les simulations ne rendent avant tout bien compte que des comportements de la croissance aérienne. En fait, nous nous trouvons actuellement dans une situation où des informations sur les systèmes racinaires seraient bien utiles.

Trois exemples:

- Les modèles classiques n'utilisant et ne délivrant que des informations dendrométriques classiques ne suffiront pas à prédire les effets des modifications prévisibles de l'environnement (notamment celles liées à l'augmentation du taux de gaz carbonique dans l'atmosphère) sur la croissance des arbres et sur la gestion des forêts. Les modèles de croissance à base écophysiologique tentent de pallier cet inconvénient. Leur développement nécessite non seulement l'intégration des connaissances actuelles sur les fonctionnements photosynthétique, hydrique et cambial des arbres mais aussi des informations sur les lois de diffusion des assimilats entre les organes de l'arbre, en particulier entre la partie aérienne et la partie racinaire.

- Les modèles de croissance du Pin maritime s'enrichissent actuellement des résultats acquis sur le matériel génétiquement amélioré. Ainsi, on observe une mauvaise corrélation juvénile-adulte pour la croissance en hauteur, qui pourrait être expliquée par des différences de migration des ressources entre racines et parties aériennes [hypothèse des "sprinters" et des "coureurs de fond" de lily (1967) et de Namkoong et Conkie (1976)]. Très schématiquement, on aurait d'une part les "sprinters" qui investiraient dans le jeune âge plus de ressources dans les parties aériennes, au détriment des racines, et d'autre part des "stayers" (capables d'un effort prolongé) qui alloueraient dans le jeune âge plus de carbone aux racines, ce qui leur permettrait de dépasser les "sprinters" lorsque la concurrence pour l'eau et les éléments minéraux devient très forte. Cette hypothèse demande à être vérifiée.

- Le Chêne, en peuplement équienne, semble ne pas trop réagir aux éclaircies pratiquées lorsque le peuplement a dépassé une hauteur dominante de $15 \mathrm{~m}$ environ. Ne pas réagir signifie ne pas pouvoir dépasser $2 \mathrm{~mm}$ de croissance annuelle sur le rayon de la grume (mesuré à $1,30 \mathrm{~m}$ ). À quoi cela est-il dû? Une façon de fournir des éléments de réponse consiste à analyser comment réagissent, après éclaircie, les compartiments concurrents que sont le houppier, le sous-étage voisin que l'on conserve pour améliorer la qualité extérieure du tronc, les racines et la grume qui sera exportée ultérieurement. Peut-on voir, sur les sections radiales des grosses racines au moins, des indications permettant de dire que ces racines ont épaissi bien plus que le tronc? Si oui, ne seraitce pas une indication d'une redistribution de la matière ligneuse au détriment du tronc?

Les données sur les systèmes racinaires sont rares car les techniques d'accès aux racines sont toujours très délicates. De nombreuses méthodes ont été mises au point parmi lesquelles:

- la méthode par explosifs (Newton et Zedaker, 1981) : la déflagration désolidarise du sol le système racinaire, que l'on tire ensuite;

- la méthode du monolithe centré sur le collet de l'arbre : on extrait du sol un volume de terre attaché aux racines, que I'on sépare ensuite (Schurman et Goedewaagen, 1971); le volume de terre que l'on peut retirer est fonction de la puissance de l'engin de terrassement;

- la méthode de la fouille (Heinrich, 1976) : on nettoie les racines en place soit dans l'ensemble du système, soit dans un seul secteur, soit une racine seulement dont on suit le cheminement centrifuge ;

- une variante de la précédente méthode est la méthode de la lucarne: on dégage la terre autour du collet en ménageant une vue très locale (DSF, 1990);

- la méthode du profil : on ouvre une tranchée soit rectiligne, soit semi-circulaire (Lévy, 1968 ; Belgrand, 1983), soit encore spirale (Huguet, 1973);

- le monolithe prélevé loin du collet : on extrait très localement un cylindre de terre par carottage, ou manuellement un petit parallélépipède; on mesure ensuite les longueurs et/ou masses de racines de différentes catégories de taille, après les avoir séparées de la terre ;

- les études en minirhizotrons (par exemple, Pagès, 1992);

- les études en conteneurs de différentes formes: 
- les suivis de croissance réalisés sur plusieurs fenêtres ménagées sur les parois de tranchées (Bédéneau et Auclair, 1989) ;

- I'utilisation de traceurs marqués pour décrire l'expansion racinaire et pour comprendre le prélèvement des éléments nutritifs (Carlier, 1987 ; Caldwell et Eissenstat, 1987).

Les rares données disponibles sur les systèmes racinaires varient suivant les objectifs ayant présidé à leur étude. Nous avons cité quelques objectifs p. 166. Les variables mesurées et le niveau de précision des mesures sont souvent différents.

Les rares données disponibles sur les systèmes racinaires sont souvent difficilement comparables, car l'expression de leur morphologie varie en fonction des combinaisons espèces-sol. D'un côté, chaque sol présente aux racines des conditions particulières d'expansion, liées à ses caractéristiques nutritionnelles et à ses propriétés physiques de pénétrabilité (par exemple, Lucot, 1990 ; Tardieu, 1990). De l'autre, chaque essence passe par des stades de développement particuliers, résultats de l'expression de plusieurs phènomènes de croissance, et caractérisés chacun par une morphologie plus ou moins typique (par exemple, Atger et Édelin, 1994). De plus, en peuplement forestier, les densités variables de plantation, le statut social, la concurrence herbacée et enfin le régime des éclaircies modèlent également la morphologie des arbres et de leurs racines.

\section{OBJECTIFS}

Les études racinaires qui débutent au sein du "programme croissance" ont pour but de répondre à des questions du type de celles données p. 166. Néanmoins nous avons vu que les méthodologies d'accès sont très nombreuses. C'est également le cas des méthodes de description puis d'analyse de la morphologie et du développement des systèmes racinaires. II convient donc d'abord, selon :

- les sols,

- les stades de développement,

- les objectifs particuliers des études,

de trouver:

- les meilleures techniques d'accès aux racines,

- les méthodologies de description et de mesure,

- et entin les méthodes d'analyse et de modélisation.

Dans le même temps, il est important de mettre en évidence les stades successifs de développement et donc de retracer la morphogénèse, de relier le mieux possible la partie racinaire à laquelle on a pu accéder à celle qui est restée invisible; et enfin de comprendre les relations de croissance radiale entre partie aérienne et système souterrain.

\section{DISPOSITIF GÉNÉRAL DE RECHERCHE}

Les études racinaires au sein du "programme croissance" ont redémarré à l'occasion du projet européen "Aspects of sustainability by afforestation of agricultural set-aside areas : development of roots and shoot/root ratios" (Maintenance durable par le reboisement des terres délaissées par l'agriculture: développement des racines et rapports tiges/racines). Ce projet est opérationnel de janvier 1994 à décembre 1997. Son objectif général est de "dégrossir la variabilité" de la morphologie racinaire en étudiant, à partir de méthodes communes de description et d'analyse (basées sur la méthode du monolithe), les systèmes racinaires de quelques essences forestières dans des sols caractéristiques des pays engagés dans le projet. II s'agit de faire ressortir les grands types de mor- 


\section{F. COLIN - F. DANJON - L. WEHRLEN}

phologie racinaire. Dans ce projet, le "programme croissance" est chargé d'étudier d'une part le Chêne sessile dans les sols bruns peu lessivés autour de Nancy, et d'autre part le Pin maritime dans les sols sableux autour de Bordeaux. Notre participation est avant tout un moyen de connaître et de mettre en commun le savoir-faire acquis par des équipes du Nord de l'Europe (Écosse, Irłande, Danemark, Allemagne) tant au plan méthodologie de collecte des données qu'au plan analyse et modélisation. Nous adapterons ce savoir-faire à nos objectifs particuliers.

\section{MÉTHODES}

\section{Méthodes d'accès aux racines}

\section{- Méthode du monolithe centré sur le collet}

Après abattage des arbres choisis, le monolithe a été sorti de terre au moyen d'une pelle mécanique montée sur chenilles, selon deux approches différentes. En forêt de Champenoux près de Nancy, où le sol est constitué de limon sur horizon pseudogley, nous avons préalablement réalisé, à l'aide d'une dent de sous-solage (figure 1, ci-dessous), un "bêchage" du monolithe à $1 \mathrm{~m}$ du collet de chaque arbre. Nous avons ensuite sorti ce monolithe en enfonçant à $1 \mathrm{~m}$ en dessous du collet un râteauendaineur qui a ressorti une motte de 1 à 2 tonnes.

À Bordeaux, où le sol est sableux, le monolithe a été sorti avec un godet de pelle mécanique en secouant le collet et en tirant verticalement le système.
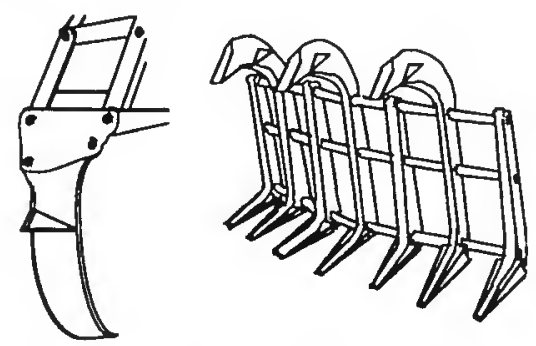

Dans les deux sites, le nettoyage a été réalisé sur le terrain soit manuellement, soit à l'aide d'un nettoyeur à eau sous haute pression, l'eau étant amenée sur le terrain par citerne.

Figure 1

DENT DE SOUS-SOLAGE (longueur de la dent $=90 \mathrm{~cm}$ ) pour bêcher autour du collet, et râteau-andaineur pour extraire le monolithe (hauteur environ $1 \mathrm{~m}$ )

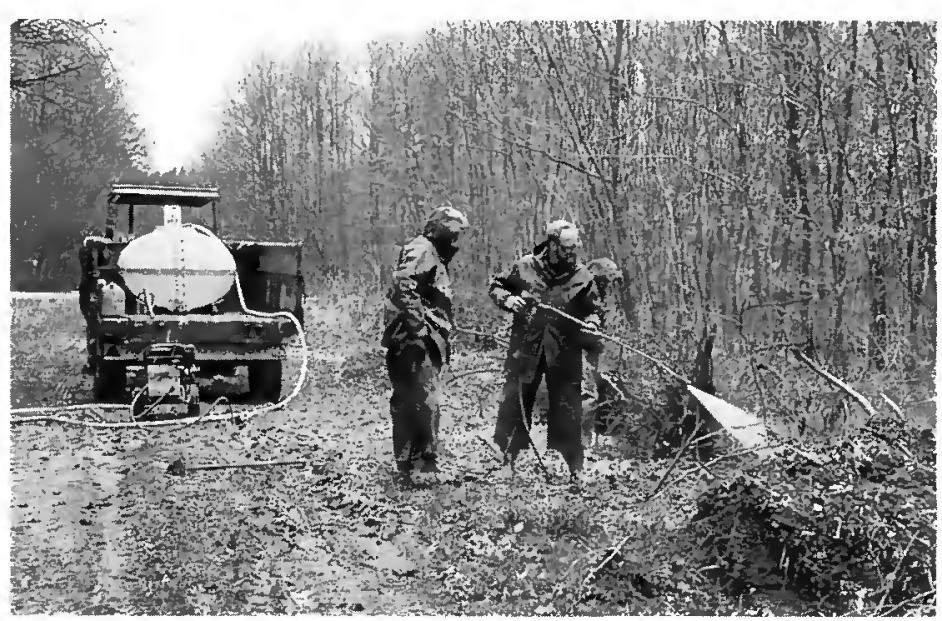

Netloyage des monolithes au nettoyeur à eau sous pression Photo $M$. PITSCH 
- Méthode du monolithe prélevé loin du collet par carottage

Au moyen d'une foreuse à moteur, nous prélevons dans les peuplements de Champenoux des carottes de terre de $10 \mathrm{~cm}$ de diamètre et de $1 \mathrm{~m}$ de longueur verticale. Les racines contenues dans ces carottes sont nettoyées. Elles sont séparées par classes de diamètre. Leur nombre, longueur et biomasse sont ensuite enregistrés. Ceci donne accès à la distribution des fines racines (à l'échelle du placeau).

\section{Utilisation d'une foreuse à mateur pour prélever des carottes de terre de $10 \mathrm{~cm}$ de diamètre et de $1 \mathrm{~m}$ de long Photo F. COLIN}

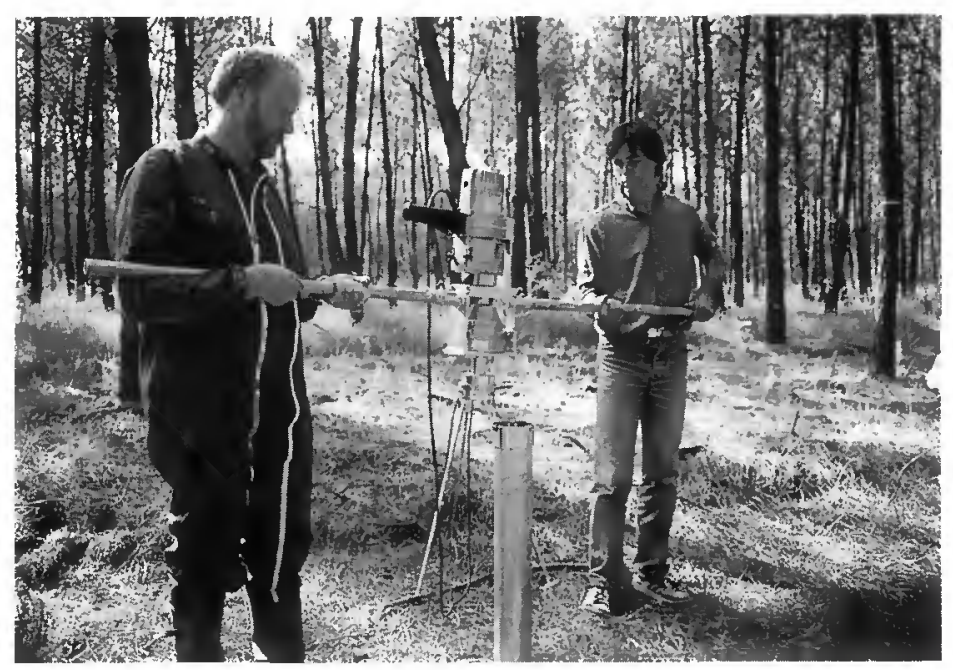

- Méthode du monolithe parallélépipédique prélevé loin du collet

Des prélèvements de racines fines ont été réalisés à Bordeaux en tranches de $15 \mathrm{~cm}$ sur 12 fosses de $40 \times 40 \mathrm{~cm}^{2}$ et de $1 \mathrm{~m}$ de profondeur. Les racines de plus de $1 \mathrm{~mm}$ ont été ventilées en cinq classes de diamètre et par espèce (Pin maritime, Molinie, Fougère). Ceci donne accès à une évaluation des interpénétrations des systèmes racinaires d'espèces concurrentes (également à l'échelle du placeau).

\section{Méthodes de description des racines issues de monolithe}

Cette méthode a été formalisée par C.N. Nielsen, coordinateur du projet européen.

Le système racinaire, une tois nettoyé, est retourné sur un plateau de mesure (tigure 2, p. 170) qui comprend un disque de contre-plaqué, quatre tasseaux et trois réglettes. Les quatre tasseaux constituent 4 rayons d'un cercle de $2 \mathrm{~m}$ de diamètre. La périphérie du disque est un cercle de $1,20 \mathrm{~m}$ de diamètre. Sur le disque est tracé un cercle concentrique de $60 \mathrm{~cm}$ de diamètre et tous les 15 grades, un rayon. Perpendiculairement à ce plateau sont fixées les trois réglettes de 40,60 et $90 \mathrm{~cm}$ de longueur.

Ce plateau de mesure permet :

— de repérer dans l'espace trois volumes cylindriques de même axe vertical ;

- d'orienter les axes des racines (azimut).

Les racines "traversent" les surfaces périphériques de ces volumes en des points qui sont les points de mesure. Y sont notés : l'orientation dans le plan horizontal (azimut), l'angle dans le plan vertical, les diamètres vertical et horizontal, et l'état sanitaire de la racine. 
À propos d'état sanitaire, on notera que ce système de description a été utilisé avec profit par l'équipe "pathologie" de I'INRA-Nancy (Cecconi, 1995).

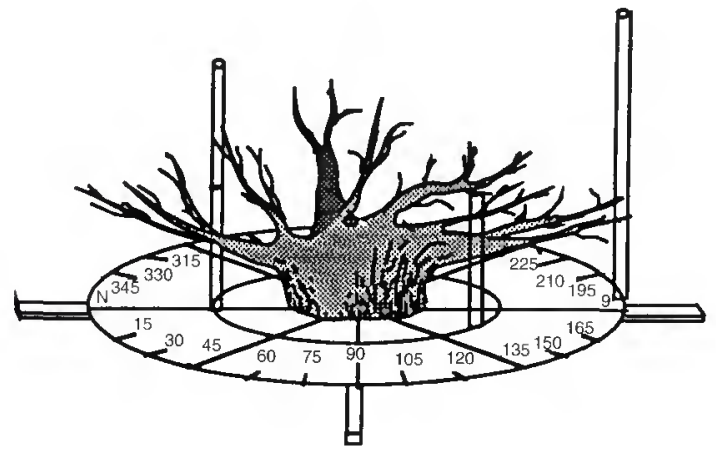

Figure 2

SYSTÈME RACINAIRE

RETDURNÉ SUR LE PLATEAU DE MESURE

\section{Méthodes d'analyse}

À partir des données recueillies selon le protocole précédent, nous cherchons à quantifier les variables suivantes:

- la biomasse aérienne (au niveau arbre),

- la biomasse de fines racines (au niveau placeau),

- le rapport biomasse aérienne - biomasse racinaire,

- la profondeur d'enracinement,

- la symétrie du système racinaire,

- les biomasses selon les différentes directions et profondeurs de sol,

- les ordres de ramification [le Chêne développe d'abord un pivot (axe d'ordre 1), à partir duquel se développent latéralement des racines traçantes (axes d'ordre 2), sur lesquelles peuvent se développer verticalement des pivots secondaires (axes d'ordre 3)],

- les modes de ramification selon les diffèrents types d'axes racinaires.

Ces variables sont ensuite comparées entre espèces et sols.

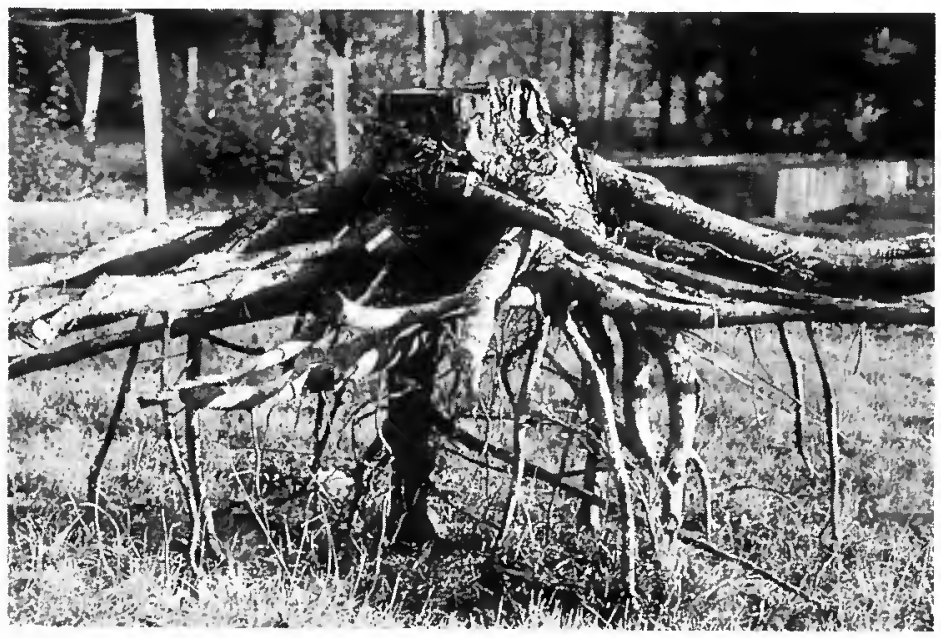

Système racinaire de Pin maritime présentant des racines horizontales, un pivol central et des pivots secondaires sous les racines horizontales

Photo F. COLIN 


\section{CONCLUSIONS}

Les études racinaires qui débutent dans le "programme croissance" ont des objectifs bien précis. Les méthodologies sont à perfectionner à partir de ce qui existe déjà, notamment à partir du système de mesure préconisé dans le projet européen qui démarre. Les essences étudiées en priorité pour l'instant sont le Chêne et le Pin maritime. Une fois les méthodes bien au point et les premiers résultats jugés intéressants, nous prendrons également en charge l'étude du Hêtre, et aborderons les essences en mélange.

F. COLIN - L. WEHALEN

Unité Croissance, Production et Qualité des bois

INRA

F-54280 CHAMPENOUX

\section{F. DANJON}

Ėquipe Croissance et Production

INRA

BP 45

PIERROTON

F-33611 GAZINET CEDEX

\section{BIBLIOGRAPHIE}

ATGER (C.), ÉDELIN (C.). - Stratégies d'occupation du milieu souterrain par les systèmes racinaires des arbres. Revue d'Écologie (Terre Vie), vol. 49, 1994, pp. 343-356.

BÉDÉNEAU (M.), AUCLAIR (D.). - The study of tree fine root distribution and dynamics using a combined trench and observation window method. - Annales des Sciences forestières, vol. 46, 1989, pp. 283-290.

BELGRAND (M.). - Comportement de jeunes plants feuillus (Chêne pédonculé, Chêne rouge, Chêne sessile, Hêtre) sur substrat ennoyé. Adaptations racinaires. Application à la mise en valeur forestiere des pseudogleys. — INA-PG, 1983. - $188 \mathrm{p}$. (Thèse).

BRÉDA (N.), COCHARD (H.), DREYER (E.), GRANIER (A.). - Water transfer in a mature oak stand (Quercus petraea) : seasonal evolution and effects of a severe drought. - Canadian Journal of Forest Research, vol, 23, 1993, pp. 11361143.

CALDWELL (M.M.), EISSENSTAT (D.M.). - Coping with variability : examples of tracer use in root function studies. In: Plant Response to Stress/J.D. Tenhunen et al. Eds. - Berlin: Springer Verlag, 1987. — pp. $95-105$ (NATO ASI Series; vol. G15).

CARLIER (G.). - Étude de la sectorisation des souches de Châtaignier (Castanea sativa Mill.) à l'aide d'eau tritiée. Annales des Sciences forestieres, vol. 44, $\mathrm{n}^{\circ} 1,1987$, pp. 85-102.

CECCONI (F.). - Collybia fusipes: quantification de la maladie dans les systèmes racinaires et relation avec la croissance radiale des arbres. - Nancy, 1995. - 23 p. (DEA Biologie forestière)

COLIN-BELGRAND (M.), JOHANNES (H.), DREYER (E.), PAGĖS (L.). - A new data processing system for root growth and ramification analysis: description of methods. - Annales des Sciences forestières, vol. 46 supp. "Forest tree Physiology"/E. Dreyer et al. Eds, 1989, pp. 305-309.

DÉPARTEMENT DE LA SANTÉ DES FORÊTS DU NORD-EST (DSF). - Méthodologie d'approche du dépérissement. Diagnostic racinaire. - Nancy : DSF, 1989. - $4 \mathrm{p}$. 


\section{F. COLIN - F. DANJON - L. WEHRLEN}

GUILLAUMIN (J.-J.), BERNARD (Ch.), DELATOUR (C.), BELGRAND (M.). — Contribution à l'étude du dépérissement du Chêne: pathologie racinaire en forêt de Tronçais. - Annales des Sciences forestières, vol. 42, $n^{\circ} 1,1985$, pp. 1-22.

HUGUET (J.-G.). - Nouvelle méthode d'étude de l'enracinement des végétaux pérennes à partir d'une tranchée spirale. - Annales agronomiques, vol. 24, $n^{\circ} 6,1973$, pp. 707-731.

ILLY (G.). - Recherches sur l'amélioration génétique du Pin maritime. - Annales des Sciences forestières, vol. $23, n^{\circ} 4$ 1967, pp. 769-948.

LÉVY (G.). - Importance des propriétés du sol pour l'enracinement de Picea excelsa et de Pinus silvestris. - Annales des Sciences forestières, vol. 25, $\mathrm{n}^{\circ} 3,1968$, pp. 157-188.

LUCOT $(E$.$) . - Première approche relative à l'interaction enracinement-pierrosité (Abies alba, Quercus petraea et Quercus$ robur'). Proposition d'un indice d'obstacle appliqué aux sols forestiers jurassiens. - Besançon : Université de FrancheComté, 1990. - 60 p. (DEA de pédologie Science des Sols).

NAMKOONG (G.), CONKLE (M.T.). - Time trends in genetic control of height growth in Ponderosa pine. - Forest Science, vol. 22, $n^{\circ} 1,1976, \mathrm{pp} .2-12$.

NEWTON (M.), ZEDAKER (S.M.). - Excavating roots with explosives. - Corvallis (USA) : Oregon State University, School of Forestry, 1981. - 5 p. (Forest Research Laboratory, Research note 68).

PAGĖS (L.). - Mini-rhizotrons transparents pour l'étude du système racinaire de jeunes plantes. Application à la caractérisation du développement racinaire de jeunes chênes (Quercus robur). - Canadian Journal of Botany, vol 70, 1992, pp. 1840-1847.

RIEDACKER (A.). - Étude de la déviation des racines horizontales ou obliques issues de boutures de Peuplier qui rencontrent un obstacle: applications pour la conception de conteneurs. - Annales des Sciences forestieres, vol. 35, $\mathrm{n}^{\circ}$ 1, 1978, pp. 1-18.

SCHURMAN (J.J.), GOEDEWAAGEN (M.A.J.). - Methods for the examination of root systems and roots. - Wageningen Centre for agricultural publishing and documentation, $197 \uparrow .-87 \mathrm{p}$.

TARDIEU (F.). - Effets de l'état structural du sol sur l'enracinement. Que prendre en compte pour la modélisation ? In : La structure du sol et son évolution/J. Boiffin et A. Marin Laflèche Éd. - Paris: INRA, 1990. - pp. 91-109. 